\title{
Biochemical effectiveness of allopurinol and allopurinol- probenecid in previously benzbromarone-treated gout patients
}

\author{
Mattheus K. Reinders • Eric N. van Roon • \\ Pieternella M. Houtman • Jacobus R. B. J. Brouwers • \\ Tim L. Th. A. Jansen
}

Received: 2 July 2006 /Revised: 14 December 2006 / Accepted: 23 December 2006 / Published online: 17 February 2007

(C) Clinical Rheumatology 2007

\begin{abstract}
In 2003, the uricosuric drug benzbromarone was withdrawn from the market. The first alternative drug of choice was the xanthine oxidase inhibitor allopurinol. The purpose was to (1) investigate the efficacy of allopurinol (standard dosage) compared with previous treatment with benzbromarone; and (2) investigate the combination therapy allopurinol-probenecid as an effective alternative treatment compared with previous benzbromarone treatment. A prospective, open study was carried out in a cohort of 51 gout patients who discontinued benzbromarone therapy because of market withdrawal. Patients were given 200-300 mg allopurinol (stage 1). When allopurinol failed to attain the target serum urate (sUr) levels $\leq 0.30 \mathrm{mmol} / \mathrm{l}$, probenecid $1,000 \mathrm{mg} /$ day was added (stage 2). Treatment with benzbromarone monotherapy (range: 100-200 mg/day; mean $138 \mathrm{mg}$ /day) resulted in $92 \%$ of patients reaching target levels $\mathrm{sUr} \leq 0.30 \mathrm{mmol} / 1$ with a decrease of $61[11] \%$ compared to baseline. In stage 1, 32 patients completed treatment with allopurinol monotherapy (range 200$300 \mathrm{mg}$ /day; mean $256 \mathrm{mg} /$ day), which resulted in $25 \%$
\end{abstract}

\footnotetext{
M. K. Reinders $(\bowtie) \cdot$ E. N. van Roon · J. R. B. J. Brouwers Department of Clinical Pharmacy and Pharmacology,

Medical Centre Leeuwarden,

Henri Dunantweg 2,

8934 AD Leeuwarden, The Netherlands

e-mail: m.reinders@znb.nl
}

M. K. Reinders • E. N. van Roon · J. R. B. J. Brouwers Department of Pharmacotherapy and Pharmaceutical Care, Groningen Research Institute for Pharmacy,

University of Groningen,

Groningen, The Netherlands

P. M. Houtman • T. L. T. A. Jansen Department of Rheumatology, Medical Centre Leeuwarden, Leeuwarden, The Netherlands of patients attaining sUr target levels. Decrease in sUr levels was $36[11] \%$, which was significantly less compared to treatment with benzbromarone $(p<0.001)$. In stage 2,14 patients received allopurinol-probenecid combination therapy, which resulted in $86 \%$ of patients attaining target sUr levels (after failure on allopurinol monotherapy), which was comparable to previous treatment with benzbromarone $(p=0.81)$. Decrease in sUr levels was 53[9]\% (CI 95\%: $48-58 \%$ ), which was a non-significant difference compared to previous treatment with benzbromarone ( $p=$ 0.23 ). Benzbromarone is a very effective antihyperuricemic drug with $91 \%$ success in attainment of target sUr levels $\leq 0.30 \mathrm{mmol} / \mathrm{l}$. Allopurinol $200-300 \mathrm{mg} /$ day was shown to be a less potent alternative for most selected patients to attain target sUr levels (13\% success). In patients failing on allopurinol monotherapy, the addition of probenecid proves to be an effective treatment strategy for attaining sUr target levels ( $86 \%$ success).

Keywords Allopurinol · Benzbromarone - Gout · Probenecid $\cdot$ Hyperuricemia

\section{Introduction}

In 2003, benzbromarone was withdrawn from the European market [1]. The reason for the withdrawal was the risk of fulminant hepatitis according to the information given by the manufacturer. Since the introduction of benzbromarone in the early 1970s, three cases of severe hepatitis have been published [2-4]. On the other hand, benzbromarone seems well tolerated in general use [5] in contrast to allopurinol, which causes frequently (2-5\%) allergic skin reactions. The uncertainty remains whether the efficacy-safety balance of 
benzbromarone really is unfavourable, compared to the other antihyperuricemic drugs available like allopurinol, probenecid and sulphinpyrazone [6]. In Europe, benzbromarone was available only as a prescription uricosuric drug in a limited number of countries. In the Netherlands, the only registered alternative for patients with chronic gout was allopurinol, a uricostatic drug acting by inhibition of xanthine oxidase.

The goal of antihyperuricemic treatment is to reduce the serum urate (sUr) level below the threshold of supersaturation to allow the dissolution of existing monosodium urate (MSUr) crystals in the joints and to stop the deposition of new crystals [7-11]. The solubility of urate in joint fluids is influenced by temperature, $\mathrm{pH}$, concentration of cations, level of articular dehydration and the presence of nucleating agents such as insoluble collagens, chondroitin sulphate and non-aggregated proteoglycans [1].

The treatment goal of antihyperuricemic therapy is usually supposed to reach sUr levels below $0.36 \mathrm{mmol} / 1$ [8-10]. It is shown that recurrent gouty attacks are better prevented
$[12,13]$ and that tophi dissolve more quickly $[14]$ in the presence of sUr levels $\leq 0.30 \mathrm{mmol} / 1$, compared with levels $0.30-0.36 \mathrm{mmol} / \mathrm{l}$. Thus, we aimed the sUr treatment goal for gout patients at $\leq 0.30 \mathrm{mmol} / \mathrm{l}$.

The amount of urate in the body depends on dietary intake, synthesis and excretion. Hyperuricemia results from the overproduction of urate $(10 \%)$, from the underexcretion of urate $(90 \%)$ or often a combination of the two [7]. Therefore, theoretically, the urate balance, in terms of the ratio of overproduction and underexcretion of urate, may be clinically important for the rational choice of a uricostatic drug, a uricosuric drug or a combination of both. However, there are no trials to support or refute this theory [6]. From observational studies, it is concluded that benzbromarone $75-120 \mathrm{mg} /$ day is very effective in the control of hyperuricemia, better than allopurinol 300 $450 \mathrm{mg} /$ day $[5,15,16]$.

We carried out an observational study to evaluate the effectiveness of allopurinol monotherapy and combination therapy of allopurinol with the uricosuric drug probenecid
Fig. 1 Flow of participants through each stage of the study. GFR Glomerular filtration rate

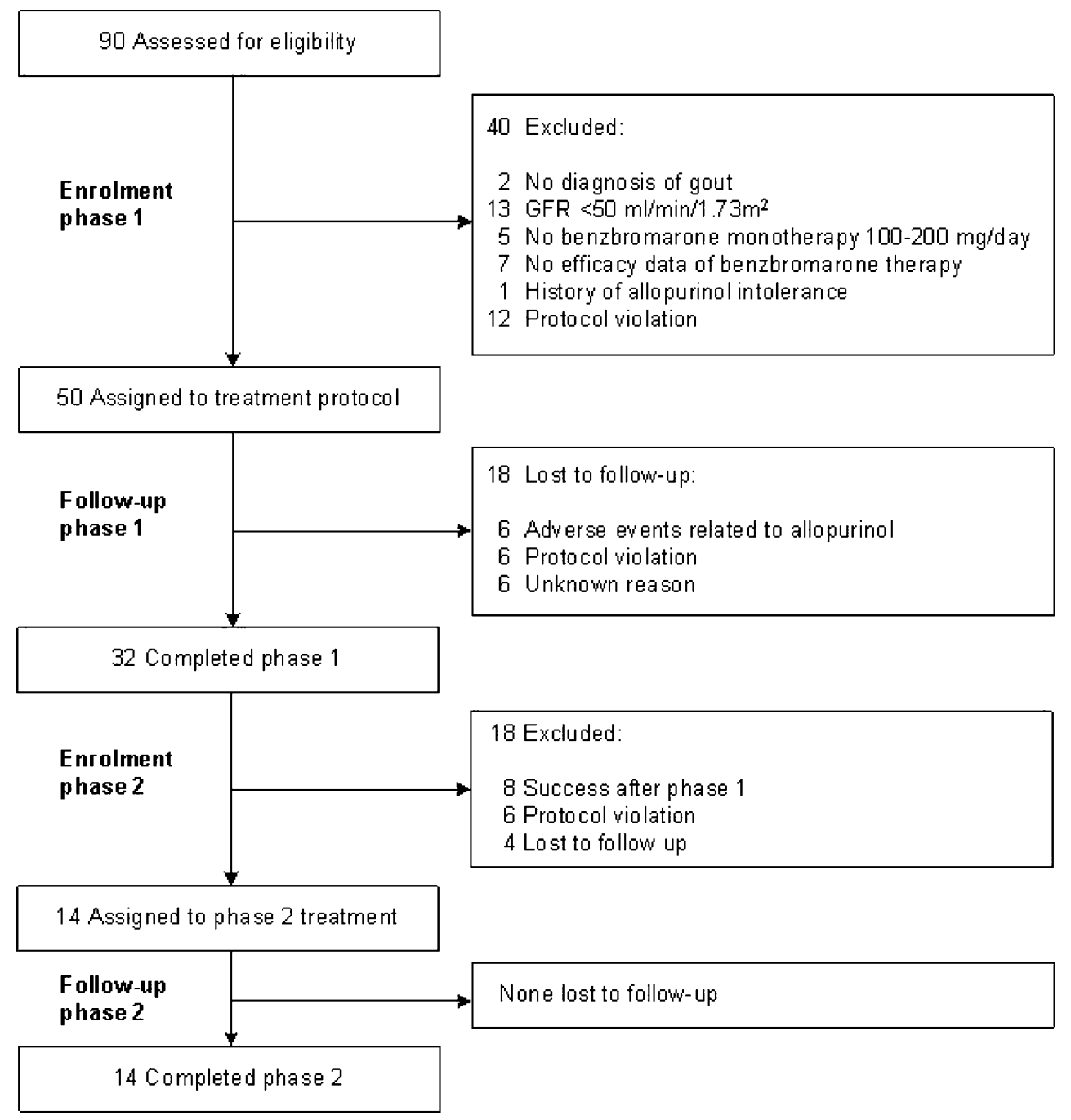


in underexcretor-type and overproducer-type gout patients previously treated with benzbromarone. Because allopurinol is reported to be less potent than benzbromarone in lowering sUr, we investigated the combination allopurinolprobenecid as an alternative treatment when allopurinol failed.

\section{Materials and methods}

A prospective, open label study was carried out in patients who had to discontinue benzbromarone therapy and contacted the rheumatology department in the period July-December 2003. The inclusion criteria were the following: (1) diagnosis of gout proven by presence of MSUr crystals in the synovial fluid [17] or otherwise complying with the American Rheumatism Association criteria [18]; (2) pre-treatment with benzbromarone monotherapy of 100-200 mg/day for at least 2 months with available sUr efficacy results; (3) no relevant liver disease and (4) no relevant renal disease, defined as glomerular filtration rate $(\mathrm{GFR})>50 \mathrm{ml} \mathrm{min}^{-1} 1.73 \mathrm{~m}^{-2}$.
Before entering the study, liver function, serum creatinine $(\mathrm{sCr})$, sUr, urinary creatinine excretion $(\mathrm{uCr})$ and urinary urate excretion ( $\mathrm{uUr}$ ) on unrestricted purine diet were measured after benzbromarone was stopped for at least 1 month. GFR was estimated from the simplified Modification of Diet in Renal Disease (MDRD) formula $[19,20]$. Underexcretion of urate was defined as a urate clearance (ratio uUr/sUr) $<6.0 \mathrm{ml} \mathrm{min}{ }^{-1} 1.73 \mathrm{~m}^{-2}$ [21]. Results of pre-treatment with benzbromarone were collected from the patient's chart.

In stage 1, patients were given allopurinol with a maximum of 200-300 $\mathrm{mg}$ once a day in a step-up dosage scheme; maximum dosage prescribed depended on kidney function, tolerability and prescriber's preference. When the treatment goal of sUr $\leq 0.30 \mathrm{mmol} / \mathrm{l}$ was not reached with allopurinol after 2 months, patients were included in stage 2 , and probenecid $500 \mathrm{mg}$ twice daily was added to allopurinol. Each treatment regimen was evaluated after a treatment period of $>2$ months, and $\mathrm{sCr}, \mathrm{sUr}, \mathrm{uCr}$ and $\mathrm{uUr}$ were measured. Prophylaxis of gouty episodes with colchicine was prescribed to all patients until target sUr level was reached $(\mathrm{sUr} \leq 0.30 \mathrm{mmol} / \mathrm{l})$.

Table 1 Demographic data, baseline urate characteristics and previous results attained with benzbromarone of patients enrolled in stage 1 (allopurinol treatment) and enrolled in stage 2 (allopurinol-probenecid treatment)

\begin{tabular}{|c|c|c|}
\hline & $\begin{array}{l}\text { Stage } 1^{\text {a }} \\
\text { Allopurinol }(n=50)\end{array}$ & $\begin{array}{l}\text { Stage } 2^{\text {a }} \\
\text { Allopurinol-probenecid }(n=14)\end{array}$ \\
\hline \multicolumn{3}{|l|}{ Demographics } \\
\hline Age (years) & $57[9]$ & $55[8]$ \\
\hline Male gender (\%) & 94 & 93 \\
\hline Tophaceous gout (\%) & 24 & 14 \\
\hline \multicolumn{3}{|l|}{ GFR $\left(\mathrm{ml} \mathrm{min}-1.73 \mathrm{~m}^{-2}\right)$} \\
\hline $50-80(\%)$ & 57 & 50 \\
\hline$>80(\%)$ & 43 & 50 \\
\hline History of allopurinol use (\%) & 20 & 14 \\
\hline \multicolumn{3}{|l|}{ Baseline urate characteristics } \\
\hline Baseline sUr (mmol/l), range & $0.55[0.08], 0.41-0.69$ & $0.54[0.08], 0.42-0.68$ \\
\hline Baseline $u U r$ (mmol/day), range & $3.7[1.3], 1.6-6.8$ & $3.8[1.7], 1.6-6.8$ \\
\hline Baseline $\mathrm{UrCl}(\mathrm{ml} / \mathrm{min})$, range & $4.8[1.9], 1.8-11.2$ & $5.0[2.5], 1.8-10.4$ \\
\hline Underexcretor-type $(\%)$ & 95 & 92 \\
\hline \multicolumn{3}{|l|}{ Previous benzbromarone results ${ }^{\mathrm{b}}$} \\
\hline Dosage benzbromarone (mg/day) & 139 [49] & $143[51]$ \\
\hline Benzbromarone sUr $(\mathrm{mmol} / \mathrm{l})$, range & $0.21[0.07], 0.09-0.38$ & $0.23[0.06], 0.14-0.31$ \\
\hline \multicolumn{3}{|l|}{ Treatment goal reached } \\
\hline $\mathrm{sUr} \leq 0.30 \mathrm{mmol} / 1(\%)$ & 92 & 93 \\
\hline $\mathrm{sUr} \leq 0.36 \mathrm{mmol} / 1(\%)$ & 98 & 100 \\
\hline Benzbromarone $\Delta \mathrm{sUr}(\%)$ & $-61[11]$ & $-58[8]$ \\
\hline Benzbromarone uUr (mmol/day), range & $5.0[1.7], 2.4-8.5$ & $5.3[1.7], 2.4-8.0$ \\
\hline Benzbromarone $\mathrm{UrCl}(\mathrm{ml} / \mathrm{min})$, range & $16.2[4.6], 6.2-24.5$ & 16.3 [4.9], 6.2-22.2 \\
\hline
\end{tabular}

GFR Glomerular filtration rate, $s U r$ serum urate, $u U r$ urate excreted in urine, $U r C l$ urate clearance

${ }^{a}$ Data shown as mean[standard deviation] and range, unless stated otherwise

${ }^{\mathrm{b}}$ Latest results before benzbromarone withdrawal 
Table 2 Effectiveness of allopurinol (stage 1) and allopurinol-probenecid combination therapy (stage 2)

\begin{tabular}{lll}
\hline & $\begin{array}{l}\text { Stage } 1^{\mathrm{a}} \\
\text { Allopurinol }(n=32)\end{array}$ & $\begin{array}{l}\text { Stage } 2^{\mathrm{a}} \\
\text { Allopurinol-probenecid }(n=14)\end{array}$ \\
\hline Dosage allopurinol (mg/day) & $256[50]$ & $243[51]$ \\
Dosage probenecid (mg/day) & - & 1,000 \\
sUr reached (mmol/l), range & $0.36[0.07], 0.24-0.57$ & $0.25[0.04], 0.20-0.35$ \\
$\Delta \mathrm{sUr}(\%)$ from baseline & $-36[11]$ & $-53[9]$ \\
$\Delta \mathrm{sUr}(\%)$ from allopurinol monotherapy & - & $-33[10]$ \\
$\Delta \mathrm{sUr}(\%)$ from benzbromarone monotherapy & $+78[60]$ & $+19[43]$ \\
$\mathrm{uUr}(\mathrm{mmol} /$ day), range & $2.3[0.8], 0.8-4.3$ & $3.1[0.9], 1.5-4.4$ \\
UrCl (ml/min), range & $4.4[1.3], 2.3-7.7$ & $8.8[2.4], 4.5-13.9$ \\
Treatment goal reached & & 86 \\
sUr $\leq 0.30$ mmol/1 (\%) & 25 & 100 \\
sUr $\leq 0.36 \mathrm{mmol} / 1 \%)$ & 53 & \\
\hline
\end{tabular}

$s U r$ Serum urate, $u U r$ urate excreted in urine, $\mathrm{UrCl}$ urate clearance

${ }^{a}$ Data shown as mean[standard deviation] and range, unless stated otherwise
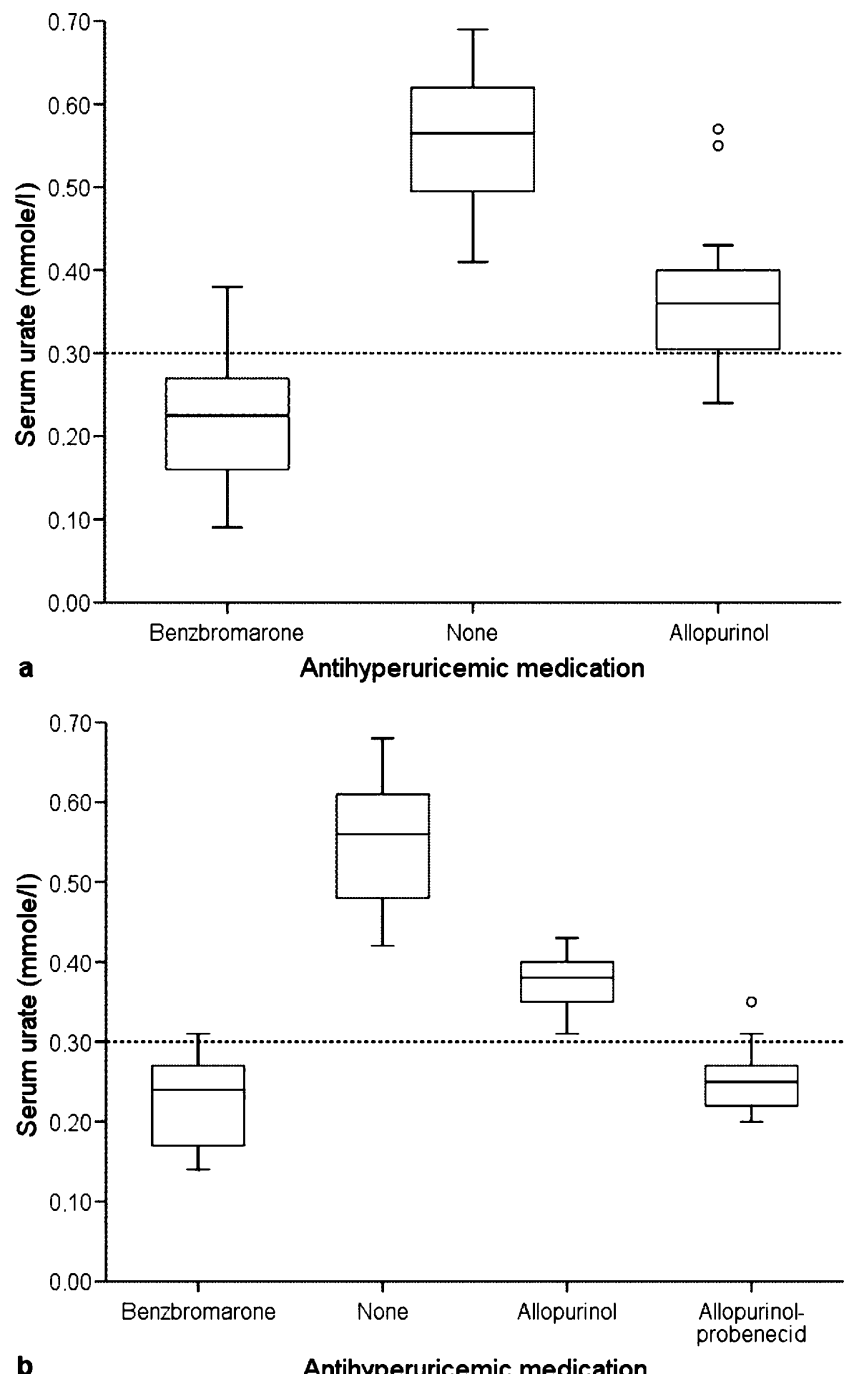

Fig. 2 Box-plot diagram for sUr results of stage 1, $n=32$ (a) and stage $2, n=14$ (b). Open circle Outlying value
Primary endpoints for stages 1 and 2 were the percentage of patients attaining sUr target $\leq 0.30 \mathrm{mmol} / 1$ and the relative decrease in $\mathrm{sUr}$ attained with each treatment regimen.

Statistical Package for the Social Sciences (SPSS) 14.0 for Windows was used for data collection, data validation, data selection and statistical analysis. Student's two-sided paired $t$ test and chi-squared test were used to compare the effectiveness of benzbromarone, allopurinol and allopurinolprobenecid therapy. Yates's continuity correction was used where appropriate. Normality was verified with Kolmogorov-Smirnov analysis. A $p$ value $<0.05$ is considered statistically significant.

\section{Results}

Ninety patients made an appointment with the rheumatology department because of benzbromarone withdrawal. Fifty patients $(55 \%)$ were assigned to stage 1 treatment and received allopurinol 200-300 mg/day (Fig. 1). Table 1 shows demographic data, urate results on benzbromarone therapy and urate results at baseline $>4$ weeks after discontinuation of benzbromarone therapy. Thirty-two patients (64\%) completed stage 1 and were included in the analysis. Eight patients attained target serum levels on allopurinol 200-300 mg/day and completed the study. Fourteen patients were assigned to and completed the combination therapy allopurinol-probenecid $500 \mathrm{mg}$ twice daily (stage 2) and were included in the analysis.

Urate results after allopurinol (stage 1) and allopurinolprobenecid (stage 2) treatment are presented in Table 2 and Fig. 2. With allopurinol monotherapy, the sUr reached was $0.36[0.07] \mathrm{mmol} / 1$, and eight (25\%) patients reached target $\mathrm{sUr} \leq 0.30 \mathrm{mmol} / \mathrm{l}$. Compared to treatment with benzbro- 
marone 100-200 mg/day, significantly less patients attained target sUr levels $(p<0.001)$. With allopurinol-probenecid, sUr reached was $0.25[0.04] \mathrm{mmol} / \mathrm{l}$, and $12(86 \%)$ patients reached target sUr after failure on allopurinol monotherapy. No significant difference was found compared to treatment with benzbromarone $(p=0.81)$.

Using allopurinol monotherapy, sUr decreased 36[11]\% compared to baseline. This was significantly less than relative sUr decrease attained with benzbromarone in this group $(p<0.001)$. With allopurinol-probenecid treatment, sUr decreased 53[9]\% (CI 95\%: 48-58\%), which did not significantly differ from benzbromarone $(p=0.23)$.

Figure 3 shows cumulative probability plots of target sUr selected and percentage of treatment success found in stages 1 and 2 for selected target sUr and different treatment regimens.

\section{Discussion}

First of all, this study shows that benzbromarone is a very potent antihyperuricemic drug; in this cohort of patients using benzbromarone 100-200 $\mathrm{mg}$ /day, more than $90 \%$ of the patients have optimal sUr levels. With allopurinol standard dosage 200-300 mg/day, comparable effectiveness cannot be achieved for this group. The average allopurinol dosage (mean $243 \mathrm{mg} /$ day) might be considered relatively low; however, (1) no efficacy data on allopurinol $\geq 600 \mathrm{mg} /$ day are available in the literature; (2) dosages $>300 \mathrm{mg} /$ day are generally not advised because of increased risk of adverse drug reactions [22]; (3) benzbromarone (mean $138 \mathrm{mg} /$ day) and probenecid (1,000 $\mathrm{mg} /$ day) could also be dosed higher.

It must be noted that every patient in this study was previously treated with benzbromarone, thereby introducing the possibility to overestimate the percentage of responders
Fig. 3 Cumulative probability plot of selected target sUr concentration and success of attainment of selected target sUr for stage $1, n=32$ (a) and stage 2, $n=14$ (b). Filled triangle Benzbromarone, filled square allopurinol, open circle allopurinol-probenecid, open square no medication, $s U r$ serum urate

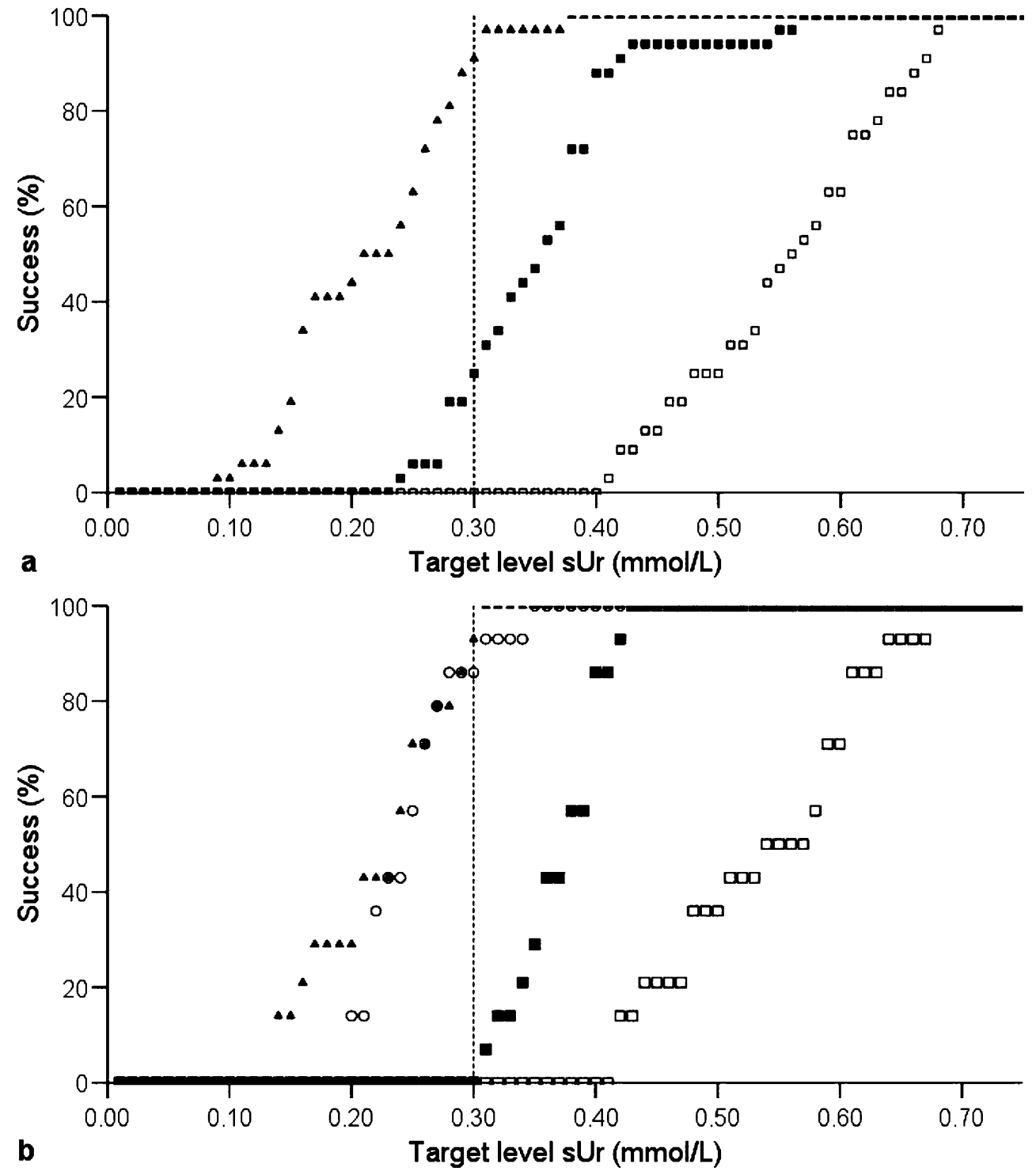


to benzbromarone. Furthermore, a dropout occurred in stage 1 of $38 \%$, which may have influenced the outcome. The relatively high dropout rate was mainly due to the design of the study (cohort) in combination with the sudden need for alternative treatment. On the other hand, we found a mean relative decrease in sUr of $61 \%$ for benzbromarone and $36 \%$ for allopurinol, which corresponds well with values published in the literature of $54-58 \%$ for benzbromarone $80-125 \mathrm{mg} /$ day and $27-44 \%$ for allopurinol $300 \mathrm{mg} /$ day in patients not previously treated with benzbromarone $[5,15,16,23,24]$.

This cohort consisted of more than $90 \%$ patients of uric acid underexcretor type. From a pathogenic point of view, it is suggested that allopurinol, as an inhibitor of uric acid production, would be more effective in overproducer-type gout. However, this is not supported by clinical data [15]. Previous findings indicate that even in patients with apparently high $u U r$, there is a relative underexcretion of urate [25]. Thus, when treatment goals are not achieved with allopurinol in this group of patients, combination with a uricosuric drug (e.g. benzbromarone, probenecid) might be very useful.

Adding probenecid to allopurinol contributed an additional 33[10]\% (CI 95\%: 28-38\%) decrease in sUr on average, resulting in reaching the target $\mathrm{sUr}$ for most patients $(87 \%$ success). Thus, in finding an appropriate alternative therapy for benzbromarone, adding probenecid to allopurinol was proven to be an effective strategy. An interaction between allopurinol and probenecid is described in the literature [26], resulting in an increased clearance of oxipurinol, the active metabolite of allopurinol. However, our data do not support this finding to be clinically important, as the addition of probenecid to allopurinol decreased sUr with $31 \%$ on average.

In stage 1, five patients (10\%) stopped allopurinol therapy because of adverse events related to allopurinol. Reported events were rash, pruritus, diarrhoea, nausea and dizziness, which are all well-known side effects of allopurinol. Becker et al. [23] found a similar rate (12\%) of adverse events related to allopurinol.

There are few therapeutic options available to lower sUr to target levels other than the drugs used in this study. The uricosuric drug sulphinpyrazone is not widely used due to its adverse effects profile [27]. Minor additive serum-lowering effects may be achieved by losartan or fenofibrate [27, 28]. The uricostatic febuxostat may be available soon. Recently, it was shown that when using febuxostat $80-120 \mathrm{mg} /$ day, $47-$ $66 \%$ of the patients reached sUr levels $\leq 0.30 \mathrm{mmol} / 1$ compared to $13 \%$ with allopurinol $300 \mathrm{mg} /$ day [23]. A new promising treatment option for patients with severe tophaceous gout is the development of recombinant uricase [29, 30] and pegylated recombinant uricase. Uricase-based drugs are potentially very effective but also very expensive drugs, so further (pharmacoeconomic) studies on optimizing antihyper- uricemic therapy with old (out of patent) drugs, like benzbromarone, are warranted. At this moment, benzbromarone seems to be the most effective antihyperuricemic drug, and from our point of view, availability of benzbromarone in other countries would make treatment of difficult gout more successful [31].

Acknowledgment The authors thank E.N. Griep, MD, Ph.D. and J. P.L. Spoorenberg, MD, Ph.D. (Medical Centre Leeuwarden) for inclusion of their patients in the study. This work has not been supported by any grant either from the pharmaceutical industry, private or public institutions.

\section{References}

1. Jansen TL, Reinders MK, van Roon EN et al (2004) Benzbromarone withdrawn from the European market: another case of "absence of evidence is evidence of absence"? Clin Exp Rheumatol 22:651

2. Van der Klauw MM, Houtman PM, Stricker BH et al (1994) Hepatic injury caused by benzbromarone. J Hepatol 20:376-379

3. Wagayama H, Shiraki K, Sugimoto K et al (2000) Fatal fulminant hepatic failure associated with benzbromarone. J Hepatol 32:874

4. Arai M, Yokosuka O, Fujiwara K et al (2002) Fulminant hepatic failure associated with benzbromarone treatment: a case report. J Gastroenterol Hepatol 17:625-626

5. Masbernard A, Giudicelli CP (1981) Ten years' experience with benzbromarone in the management of gout and hyperuricaemia. $\mathrm{S}$ Afr Med J 59:701-706

6. Sutaria S, Katbamna R, Underwood M (2006) Effectiveness of interventions for the treatment of acute and prevention of recurrent gout - a systematic review. Rheumatology 45:1422-1431

7. Choi KH, Mount DB, Reginato AM (2005) Pathogenesis of gout. Ann Intern Med 143:499-516

8. Terkeltaub RA (2003) Gout. N Engl Med J 349:1647-1655

9. Rott KT, Agudelo CA (2003) Gout. JAMA 289:2857-2860

10. Wortmann RL (2005) Recent advances in the management of gout and hyperuricemia. Curr Opin Rheumatol 17:319-324

11. Li-Yu J, Clayburne G, Sieck M et al (2001) Treatment of chronic gout. Can we determine when urate stores are depleted enough to prevent attacks of gout? J Rheumatol 28:577-580

12. Shoji A, Yamanaka H, Kamatani N (2004) A retrospective study of the relationship between serum urate level and recurrent attacks of gouty arthritis: evidence for reduction of recurrent gouty arthritis with antihyperuricemic therapy. Arthritis Rheum 51:321-325

13. Yamanaka H, Togashi R, Hakoda M et al (1998) Optimal range of serum urate concentrations to minimize risk of gouty attacks during anti-hyperuricemic treatment. Adv Exp Med Biol 431:13-18

14. Perez-Ruiz F, Calabozo M, Pijoan JI et al (2002) Effect of uratelowering therapy on the velocity of size reduction of tophi in chronic gout. Arthritis Rheum 47:356-360

15. Perez-Ruiz F, Alonso-Ruiz A, Calabozo M et al (1998) Efficacy of allopurinol and benzbromarone for the control of hyperuricaemia. A pathogenic approach to the treatment of primary chronic gout. Ann Rheum Dis 57:545-549

16. Hanvivadhanakul P, Akkasilpa S, Deesomchok U (2002) Efficacy of benzbromarone compared to allopurinol in lowering serum urate level in hyperuricemic patients. J Med Assoc Thai 85:S40-S47

17. Pascual E, Batlle-Gualda E, Martinez A et al (1999) Synovial fluid analysis for diagnosis of intercritical gout. Ann Intern Med $131: 756-759$ 
18. Wallace SL, Robinson H, Masi AT et al (1977) Preliminary criteria for the classification of the acute arthritis of primary gout. Arthritis Rheum 20:895-900

19. Traynor J, Mactier R, Geddes CC et al (2006) How to measure renal function in clinical practice. BMJ 333:733-737

20. Levey AS, Greene T, Kusek JW et al (2000) A simplified equation to predict glomerular filtration rate from serum creatinine. J Am Soc Nephrol 11:A0828

21. Kaehny WD, Tangel DJ, Johnson AM et al (1990) Uric acid handling in autosomal dominant polycystic kidney disease with normal filtration rates. Am J Med 89:49-52

22. Hande KR, Noone RM, Stone WJ (1984) Severe allopurinol toxicity. Description and guidelines for prevention in patients with renal insufficiency. Am J Med 76:47-56

23. Becker MA, Schumacher HR Jr, Wortmann RL et al (2005) Febuxostat compared with allopurinol in patients with hyperuricemia and gout. N Engl J Med 353:2450-2461

24. Sarawate CA, Patel PA, Schumacher HR et al (2006) Serum urate levels and gout flares: analysis from managed care data. J Clin Rheumatol 12:61-65
25. Perez-Ruiz F, Calabozo M, Erauskin GG et al (2002) Renal underexcretion of uric acid is present in patients with apparent high urinary uric acid output. Arthritis Rheum 47:610-613

26. Yamamoto T, Moriwaki Y, Takahashi S et al (1991) Effects of pyrazinamide, probenecid, and benzbromarone on renal excretion of oxypurinol. Ann Rheum Dis 50:631-633

27. Schlesinger N (2004) Management of acute and chronic gouty arthritis: present state-of-the-art. Drugs 64:2399-2416

28. Takahashi S, Moriwaki Y, Yamamoto T et al (2003) Effects of combination treatment using anti-hyperuricaemic agents with fenofibrate and/or losartan on urate metabolism. Ann Rheum Dis $62: 572-575$

29. Vogt B (2005) Urate oxidase (rasburicase) for treatment of severe tophaceous gout. Nephrol Dial Transplant 20:431-433

30. Moolenburgh JD, Reinders MK, Jansen TL (2006) Rasburicase treatment in severe tophaceous gout; a novel therapeutic option. Clin Rheumatol 25:749-752

31. Fam AG (2001) Difficult gout and new approaches for control of hyperuricemia in the allopurinol-allergic patient. Curr Rheumatol Rep 3:29-35 